\title{
Rapid Intervention Through Telephone Encounters for Blood Pressure Control by Residents in an Outpatient Resident Clinic
}

\author{
Basil Verghese ${ }^{1}$, Sanjana K. Kashinath ${ }^{2}$, Sonam Kiwalkar ${ }^{3}$, Christopher M. Henderson ${ }^{1}$ \\ 1. Medicine, Rochester General Hospital, Rochester, USA 2. Internal Medicine, Rochester General Hospital, Rochester, \\ USA 3. Rheumatology, Oregon State Health University, Portland, USA
}

Corresponding author: Basil Verghese, drbasilgeorge@gmail.com

\section{Abstract \\ Introduction}

Sixty-seven million Americans have hypertension that costs the nation $\$ 47.5$ billion each year. The aim of this study was to determine if regular phone calls by residents helped achieve better blood pressure control.

\section{Methods}

The study was a randomized open-labeled study in a resident-run outpatient clinic in Rochester, New York. A total of 57 poorly controlled hypertensives in the clinic were divided into two groups. All the patients received scheduled phone calls once every two weeks for a total of 24 weeks. In one group, the medications were adjusted over the phone and the other group was referred to be seen in the clinic for elevated blood pressures. Both the groups were compared to the usual standard of care group.

\section{Results}

Fifty-eight patients were recruited for the trial out of which 53 were used for the final data analysis. Eleven patients completed the trial and had a mean drop of systolic blood pressure (SBP) and diastolic blood pressure (DBP) of 28 and $11 \mathrm{mmHg}$ with $\mathrm{p}<0.01$ and $\mathrm{p}<0.03$, respectively. Among the patients who did not complete the trial but answered at least one phone call, the mean drop of SBP and DBP was 29 and $8 \mathrm{mmHg}$ with a $\mathrm{p}<0.001$ and $\mathrm{p}<0.008$, respectively. When these were compared to the usual standard of care group, the mean drop in SBP was 28.36 (12.36-48.36), 29.85 (11.85-47.85), and $0.76(8.04-9.56)$ with a $p<0.02$.

\section{Conclusions}

Patients enrolled in the trial had much better blood pressure control compared to the usual standard of care. Residents can take greater ownership of patients to help achieve better blood pressure control. To our knowledge this is the first such study done exclusively by residents in a resident-run clinic.

Received 07/26/2018

Review began 07/31/2018

Review ended 08/02/2018

Published 08/08/2018

๑) Copyright 2018

Verghese et al. This is an open access article distributed under the terms of the Creative Commons Attribution License CC-BY 3.0., which permits unrestricted use, distribution, and reproduction in any medium, provided the original author and source are credited.
Categories: Internal Medicine, Medical Education, Quality Improvement

Keywords: hypertension, resident training, outpatient department, telephone, behavioral medicine, medication

\section{Introduction}

Seventy-five million Americans have hypertension, which costs the nation $\$ 47.5$ billion each year [1]. Studies have shown that regular phone calls by healthcare workers or telemetry monitoring can help achieve better patient compliance and blood pressure control [2-10]. Studies have also shown that blood pressures that were more rapidly controlled had a more durable effect compared to others [11]. None of these studies to our knowledge has been done in a resident-run clinic or by residents. Patients in the resident-run clinic are a different population as most of the patients are in the low socio-economic groups and many are without insurance. These patients also have poor compliance and high no-show rates in the clinic making it a challenge to follow up on chronic medical conditions like hypertension and diabetes [12]. The aim of the study was to determine if regular phone calls by residents in a resident-run clinic to patients with poorly controlled hypertension with or without adjustments of oral antihypertensive medications over the phone could help achieve better blood pressure control more rapidly compared to the usual standard of care.

\section{Materials And Methods}

The Rapid Intervention through Telephone Encounters for Blood Pressure control (RITE-BP) trial was a prospective, randomized, open-labeled trial that was conducted in the resident-run outpatient medicine clinic at the Rochester General Hospital, Rochester, New York. The study protocol was approved by the institutional review board at our hospital. 
Patients were identified and recruited between March 2015 and October 2015. Patients were randomly selected and approached for enrolment when they visited the clinic for a follow up appointment. The inclusion and exclusion criteria were as outlined below.

\section{Inclusion criteria}

- Systolic blood pressure (SBP) of $\geqslant 150$ and/or diastolic blood pressure (DBP) of $\geqslant 90$ as documented in two or more consecutive previous visits to the clinic.

- $\quad$ Age 25-70 years.

\section{Exclusion criteria}

- If they followed with a cardiologist or a nephrologist

- If they were followed by the hypertension clinic

- $\quad$ Non-English speaking patients

- Did not have a working phone number

- Documented learning disabilities

- Pregnancy.

Once enrolled and after consent was obtained, they were randomly assigned to Group A or Group B. Patients in both the groups received a home blood pressure monitoring kit, a dietary pyramid chart indicating the foods they should be taking to help achieve better blood pressure control, and an exercise chart listing the various aerobic exercises they should try for at least 30 minutes, five times a week. The patients were asked to check their blood pressures twice a day preferably in the morning and evening and record them in a log that was provided to them. For the next six months, patients in both groups received a phone call from one of the investigators every two weeks. During each phone call the patients were asked questions from a preset questionnaire. The responses were noted, and the patients' blood pressure was averaged over a two-week period. If the averaged blood pressure was $>150$ systolic and or $>90$ diastolic in Group A, changes were made to their anti-hypertensive medication regimen after discussing with the clinic preceptor. If the averaged blood pressures were elevated in Group B, a follow up appointment was made for them with their primary care provider in the clinic. If a patient did not answer three consecutive calls over a six-week period, they were subsequently excluded from the trial. The patients in both the groups had a follow up appointment with one of the investigators at three months and at completion of the trial at six months at which time their blood pressures were measured, logs documented, and counseling provided again on diet, exercise, and medication compliance (Figure 1). 

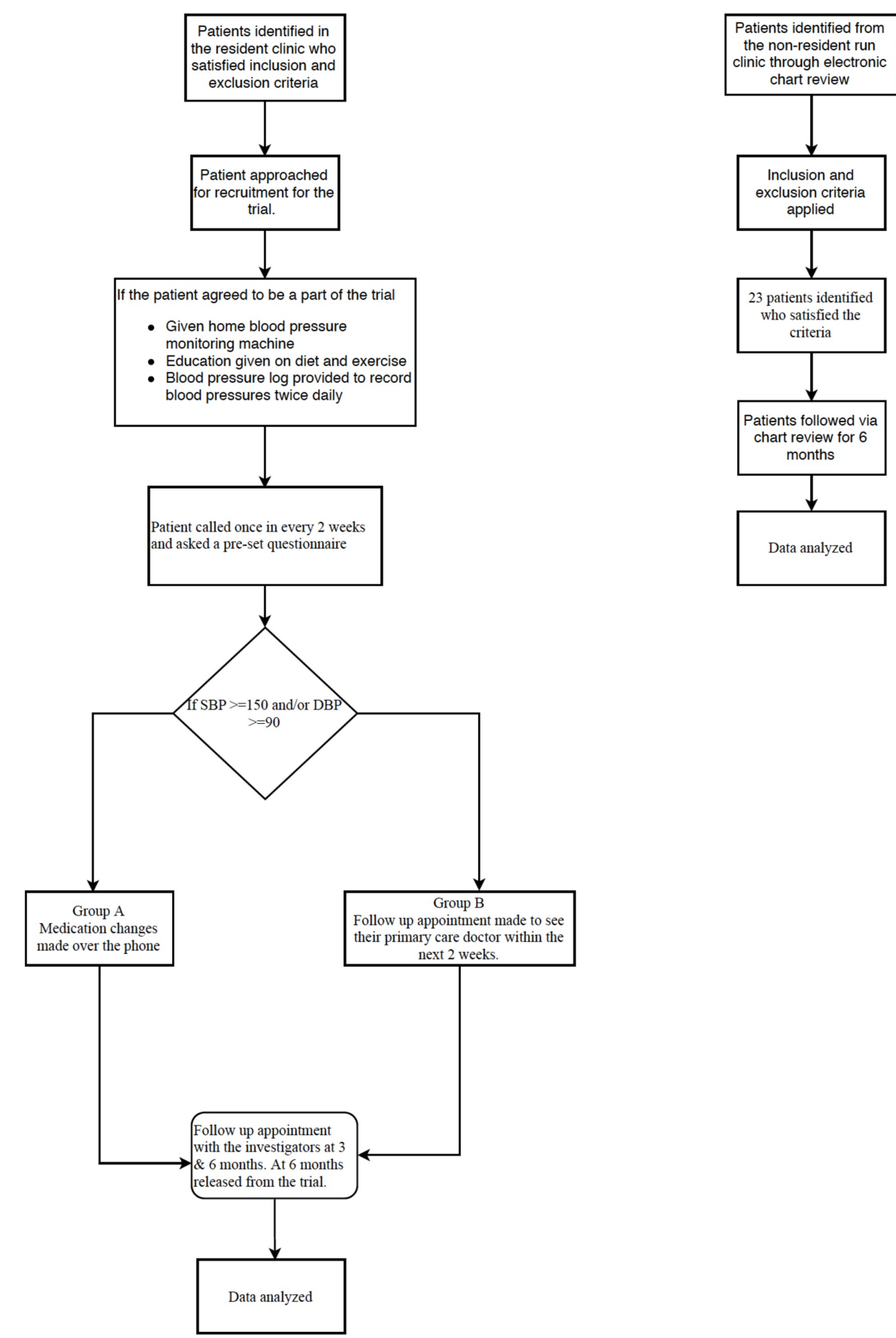

FIGURE 1: Flowchart of the study protocol.

Patients were also recruited through chart review from the nonresident run outpatient medicine clinic at the same hospital from March 2015 to October 2015 who fulfilled the above inclusion and exclusion criteria and were followed for a six-month period. Patients in this group were labeled as Group C.

\section{Statistical analysis}

We used the $t$-test, paired $t$-test, analysis of variance (ANOVA) tests, and Fischer's exact test for data analysis. Baseline characteristics were compared between three groups of patients with ANOVA tests, Chisquare tests, and Fisher's exact test. Continuous variables are expressed as mean (M) and standard deviation (SD). Dichotomous variables are expressed as number (n) and percentage (\%). The ANOVA analysis was used to compare means for continuous variables. The Chi-square tests were used to compare proportions for categorical variables. The Fisher's exact test was used for categorical variables when one or more values in the contingency table were below five. To evaluate the efficacy of intervention, paired $t$-test was performed 


\section{Cureus}

to compare blood pressure values before and after.

\section{Results}

During the recruitment period from March 2015 to October 2015 we approached 183 patients who were eligible for the trial. Of these, 58 patients agreed to be a part of the study. Five patients were excluded from the final analysis, one patient started following with cardiology and another with nephrology for their blood pressure control; three patients did not have a documented blood pressure at the end of six months (Figure 2).

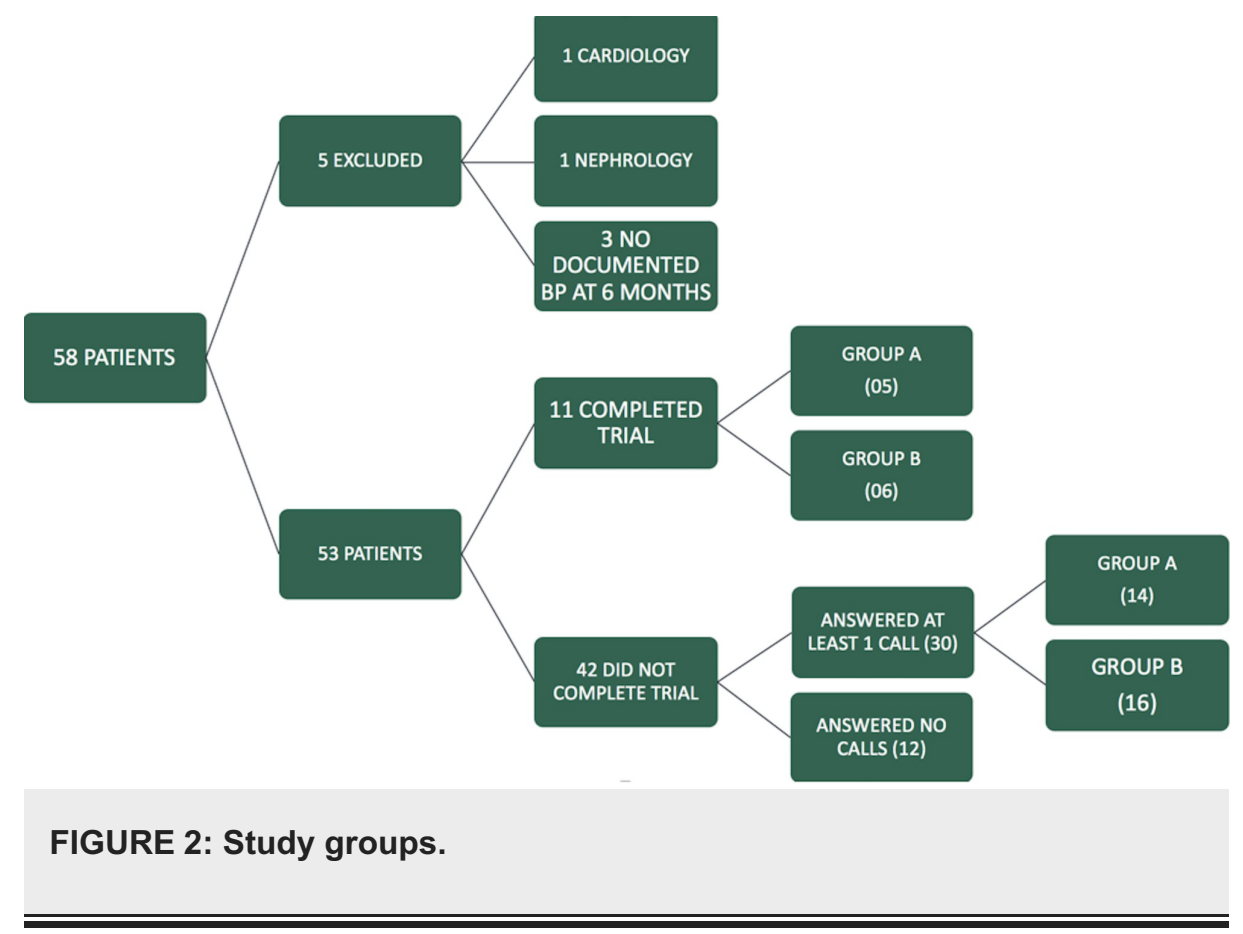

The baseline characteristics of the study population are outlined in Table 1 . 


\section{Cureus}

\begin{tabular}{|c|c|c|c|c|}
\hline \multirow[t]{2}{*}{ Baseline characteristics } & Completed trial & Not completed trial & Control Group C & $\mathbf{p}$ \\
\hline & $(n=11)$ & $(n=42)$ & $(n=21)$ & \\
\hline Mean age in years & 59.54 & 51.32 & 55 & 0.64 \\
\hline \multicolumn{5}{|l|}{ Sex } \\
\hline Male \% & 55 & 48 & 47 & 0.81 \\
\hline Female \% & 45 & 52 & 53 & \\
\hline \multicolumn{5}{|l|}{ Race } \\
\hline African American & 2 & 26 & 8 & 0.08 \\
\hline Hispanic & 3 & 8 & 8 & \\
\hline Caucasian & 6 & 8 & 5 & \\
\hline \multicolumn{5}{|l|}{ Smoking status } \\
\hline Never & 5 & 14 & 10 & 0.07 \\
\hline Former & 5 & 13 & 2 & \\
\hline Current & 1 & 15 & 9 & \\
\hline Diabetes mellitus & 2 & 21 & 3 & $<0.02$ \\
\hline LDL >100 mg/dl & 7 & 10 & 9 & 0.65 \\
\hline \multicolumn{5}{|l|}{ BMI } \\
\hline$<29$ & 1 & 11 & 8 & 0.82 \\
\hline $30-39$ & 9 & 16 & 10 & \\
\hline$>40$ & 1 & 15 & 5 & \\
\hline \multicolumn{5}{|l|}{ Systolic blood pressure } \\
\hline $150-159 \mathrm{mmHg}$ & 4 & 14 & 7 & 0.89 \\
\hline $160-169 \mathrm{mmHg}$ & 3 & 10 & 8 & \\
\hline$>170 \mathrm{mmHg}$ & 4 & 18 & 6 & \\
\hline \multicolumn{5}{|l|}{ Diastolic blood pressure } \\
\hline$<89 \mathrm{mmHg}$ & 6 & 18 & 12 & 0.06 \\
\hline$>=90 \mathrm{mmHg}$ & 5 & 24 & 9 & \\
\hline
\end{tabular}

TABLE 1: Baseline characteristics of the various study groups.

On comparison of the mean drop in SBP (in $\mathrm{mmHg}$ ) between the groups who completed the trial, those who did not complete and control Group C, we noticed a drop of 28.36 (12.36 to 48.36), 29.85 (11.85 to 47.85), and $0.76(-8.04$ to 9.56$)$ respectively which was statistically significant with $\mathrm{p}=0.02$ (Figure 3). 


\section{Cureus}

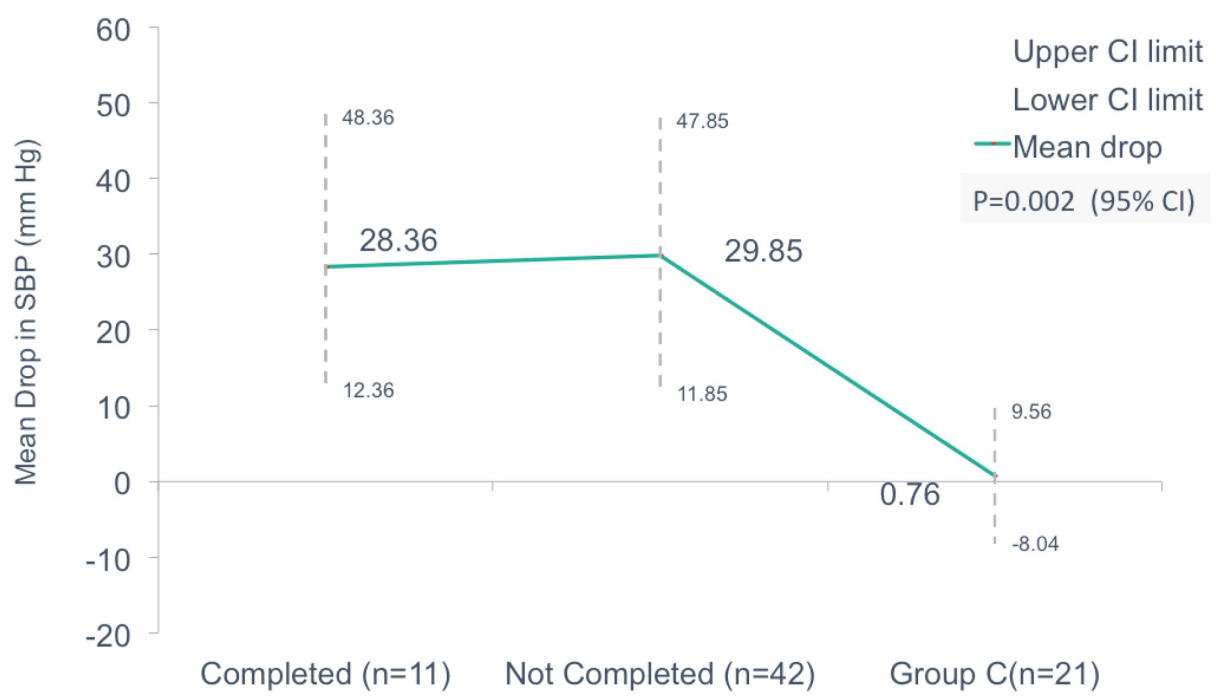

FIGURE 3: Comparison of mean drop in systolic blood pressure in those who completed the trial, did not complete the trial, and standard of care.

When we compared the mean drop in DBP (in $\mathrm{mmHg}$ ) of the same groups we found a mean drop of 11.09 (2.62 to 25.94$), 11.85$ (-5.81 to 47.85 ), and 1.19 (-14.34 to 16.43) for those who completed the trial, not completed the trial, and the control Group C, respectively, with a p $=0.051$ (Figure 4).

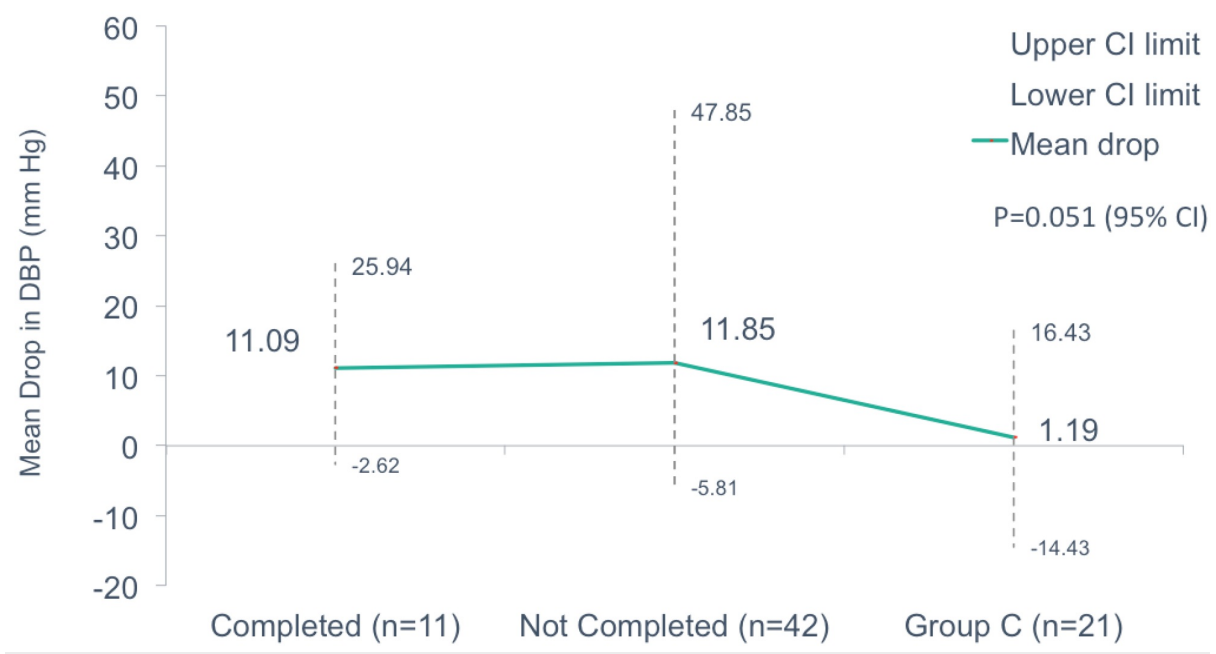

FIGURE 4: Comparison of mean drop in diastolic blood pressure in those who completed the trial, did not complete the trial, and standard of care.

We then proceeded to analyze the drop in SBP and DBP in those who did not complete the trial and those who did complete the trial. In those who did not complete the trial, the mean SBP (in mmHg) before the trial and at the completion of the trial was $170.47+/-15.25$ and $140.61+/-12.89$ with $\mathrm{p}=0.018$ and the mean DBP was $92.66+/-14.04$ and $80.8+/-13.65$ with $\mathrm{p}=0.001$, respectively. For those who completed the trial, the mean SBP (in mmHg) before and at the completion of trial was $166.18+/-13.49$ and $137.81+/-14.81$ with p = 0.0001 and mean DBP was $91.9+/-13.08$ and $80.81+/-12.68$ with $\mathrm{p}=0.0001$, respectively (Figures 5-6). 


\section{Cureus}

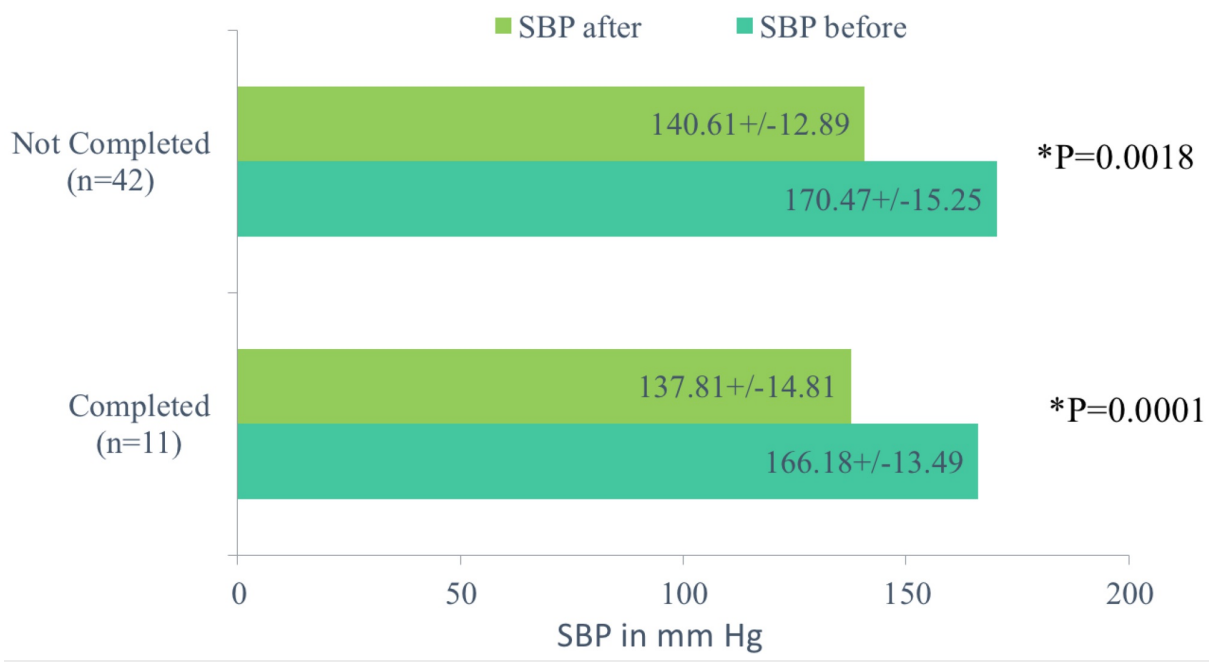

FIGURE 5: Comparison of mean systolic blood pressure in those who completed the trial and those who did not complete the trial.

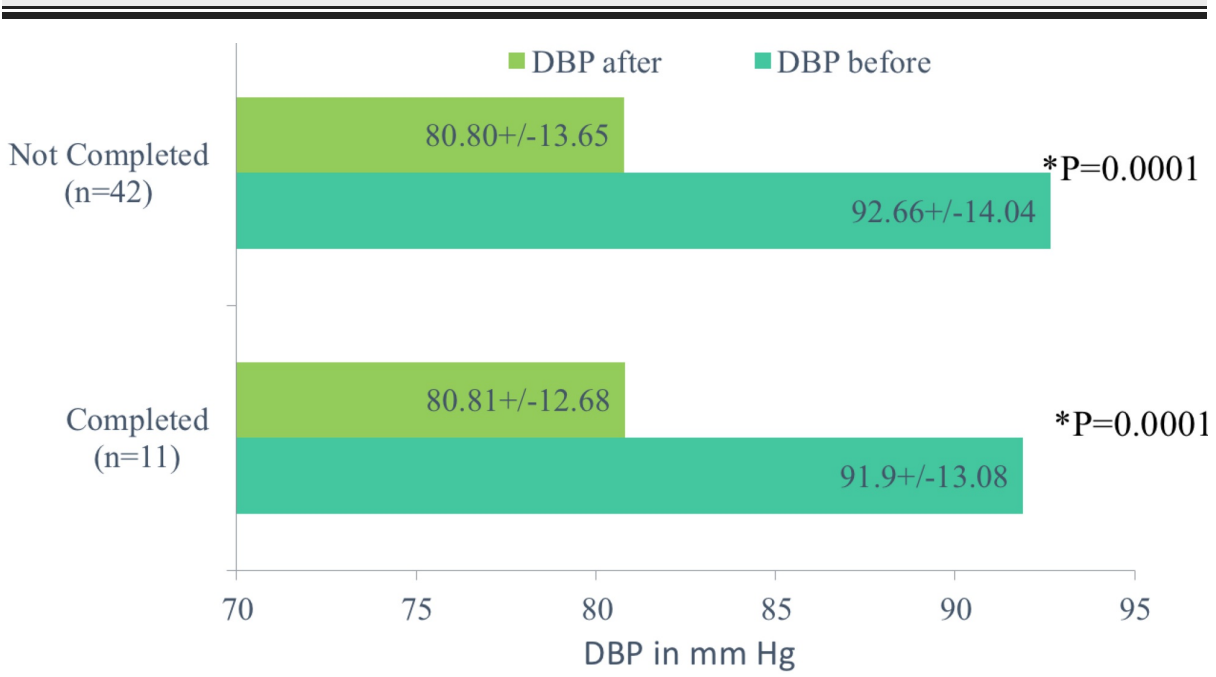

FIGURE 6: Comparison of mean diastolic blood pressure in those who completed the trial and those who did not complete the trial.

\section{Discussion}

One particular area that continues to be neglected is the resident-run clinics when it comes to blood pressure control. These resident clinic patients are usually from a low socio-economic background, diverse cultural group with no insurance or government-sponsored plans like Medicare and Medicaid [13-17]. Patients who come to the resident clinic have poor compliance, increased no-show rates (5\%-55\%), and are one of the highest risk populations [18-21].

The RITE-BP trial showed that patients who enrolled in the study, irrespective of which group they belonged to had significantly better blood pressures compared to the control group at the end of six months. The patients in the control group belonged to the same population group as the study patients with similar baseline characteristics.

Our study also showed that there was no statistical difference in blood pressures among the patients who had changes made to their medications over the phone and those who hadn't. What is even more surprising was that in the medication group only one patient's medication was changed over the phone. Over the sixmonth period of the study no medication changes were made by the regular primary care providers for the patients enrolled in the study. The common denominator for all the groups in our study population was the device given to measure their blood pressures and counseling on exercise and diet. 
exercise given at the initial visit, and knowledge that a provider was closely following their blood pressures as a psychologically motivating factor were responsible for better blood pressure values in our study subjects compared to the control group. Positive affirmation and psychology have been previously shown to be strong motivating factors to help achieve better blood pressure control [22-24].

Our study has several limitations. As medication changes were being made to the study, participants and investigators were not blinded. The study was done and data were analyzed before the results of the SPRINT trial were published, although currently the JNC VIII guidelines are still in vogue on which our study is based. Of the initial 183 patients that we approached, only 58 agreed to join our trial. One may argue that the patients who volunteered for our study were motivated enough to ensure medication, dietary, and exercise compliance which resulted in the results that we were able to achieve. Our study also had a very low number completing the trial. This is in line with generally patients having poor follow up rates at residentrun clinics and issues with compliance. The no-show rate at our clinic is around $25 \%-35 \%$. Our sample size was small. We also relied on our patients to provide accurate manual blood pressure readings, which we believe our patients did provide honestly; however, there is always a chance that they could be just giving us random numbers. But we followed up with the patients at three and six months and their logs and blood pressure readings in the clinic reflected the change just as they had said. Our follow up period was short and it would be interesting to follow these patients to see if there has been any residual effect due to our trial.

There are several future directions that the trial can take to further analyze this issue. The use of mobile phone apps has increased in recent years and the use of an algorithm to remind patients to check their blood pressures and take their medications could help. One would also like to analyze the economic impact of utilizing residents and the overall manpower required to help achieve better blood pressure control.

\section{Conclusions}

In conclusion, our trial was able to show that residents were able to achieve a significant drop in systolic and diastolic blood pressures of the patients who were motivated, received regular phone calls, had a blood pressure measuring device, and received intense counseling on exercise and dietary habits compared to the usual standard of care groups. Perhaps following these steps could help us curb this ever-growing problem.

\section{Additional Information \\ Disclosures}

Human subjects: Consent was obtained by all participants in this study. Rochester General Health System Clinical Investigation Committee issued approval CIC 1506-B-14-1. The Chair for the Rochester General Health System Clinical Investigation Committee has reviewed the information you have submitted including the protocol regarding this study and has given its approval. Animal subjects: All authors have confirmed that this study did not involve animal subjects or tissue. Conflicts of interest: In compliance with the ICMJE uniform disclosure form, all authors declare the following: Payment/services info: All authors have declared that no financial support was received from any organization for the submitted work. Financial relationships: All authors have declared that they have no financial relationships at present or within the previous three years with any organizations that might have an interest in the submitted work. Other relationships: All authors have declared that there are no other relationships or activities that could appear to have influenced the submitted work.

\section{Acknowledgements}

The authors would like to acknowledge the assistance of Dr. Ha who helped with the statistics of the study.

\section{References}

1. Mozzafarian D, Benjamin EJ, Go AS, et al.: Heart disease and stroke statistics-2015 update: a report from the American Heart Association. 2015, 131:e29-e322. 10.1161/CIR.0000000000000152

2. Parati G, Omboni S, Albini F, et al.: Home blood pressure telemonitoring improves hypertension control in general practice. The TeleBPCare study. J Hypertens. 2009, 27:198-203. 10.1097/HJH.0b013e3283163caf

3. Madsen LB, Kirkegaard P, Pedersen EB: Blood pressure control during telemonitoring of home blood pressure. A randomized controlled trial during 6 months. Blood Press. 2008, 17:78-86. 10.1080/08037050801915468

4. Møller DS, Dideriksen A, Sørensen S, Madsen LD, Pedersen EB: Tele-monitoring of home blood pressure in treated hypertensive patients. Blood Press. 2003, 12:56-62. 10.1080/08037050310000472

5. Krousel-Wood MA, Re RN, Abdoh A, et al.: Patient and physician satisfaction in a clinical study of telemedicine in a hypertensive patient population. J Telemed Telecare. 2001, 7:206-211. 10.1258/1357633011936417

6. Friedman RH, Kazis LE, Jette A: A telecommunications system for monitoring and counseling patients with hypertension. Impact on medication adherence and blood pressure control. Am J Hypertens. 1996, 9:285292. 10.1016/0895-7061(95)00353-3

7. Wang V, Smith VA, Bosworth HB, et al.: Economic evaluation of telephone self-management interventions for blood pressure control. Am Heart J. 2012, 163:980-986. 10.1016/j.ahj.2012.03.016

8. Neumann CL, Menne J, Rieken EM, et al.: Blood pressure telemonitoring is useful to achieve blood pressure 
control in inadequately treated patients with arterial hypertension. J Hum Hypertens. 2011, 25:732-738. 10.1038/jhh.2010.119

9. Jones MI, Greenfield SM, Bray EP, et al.: Patients' experiences of self-monitoring blood pressure and selftitration of medication: the TASMINH2 trial qualitative study. Br J Gen Prac. 2012, 62:135-142. 10.3399/bjgp12X625201

10. McKinstry B, Hanley J, Wild S, et al.: Telemonitoring based service redesign for the management of uncontrolled hypertension: multicentre randomised controlled trial. Br Med J. 2013, 346:3030. 10.1136/bmj.f3030

11. Datta SK, Oddone EZ, Olsen MK, et al.: Economic analysis of a tailored behavioral intervention to improve blood pressure control for primary care patients. Am Heart J. 2010, 160:257-263. 10.1016/j.ahj.2010.05.024

12. Nguyen DL, Dejesus RS, Wieland ML: Missed appointments in resident continuity clinic: patient characteristics and health care outcomes. J Grad Med Educ. 2011, 3:350-355. 10.4300/JGME-D-10-00199.1

13. MUA Find. (2018). Accessed: July 29, 2018: https://datawarehouse.hrsa.gov/tools/analyzers/muafind.aspx.

14. Tesch B, Lee H, McDonald M: Reducing the rate of missed appointments among patients new to a primary care clinic. J Ambul Care. 1984, 7:32-41.

15. Nguyen DL, Dejesus RS: Increased frequency of no-shows in residents' primary care clinic is associated with more visits to the emergency department. J Prim Care Community Health. 2010, 1:8-11. 10.1177/2150131909359930

16. Stone CA, Palmer JH, Saxby PJ, Devaraj VS: Reducing non-attendance at outpatient clinics . J R Soc Med. 1999, 92:114-118.

17. Hixon Al, Chapman RW, Nuovo J: Failure to keep clinic appointments: implications for residency education and productivity. Fam Med. 1999, 31:627-630.

18. Johnson BJ, James MW, Pontious MJ: Reduction and management of no-shows by family medicine residency practice exemplars. Ann Fam Med. 2007, 5:534-538. 10.1370/afm.752

19. Weingarten N, Meyer DL, Schneid JA: Failed appointments in residency practices: who misses them and what providers are affected. J Am Board Fam. 1997, 10:407-411.

20. James PA, Oparil S, Carter BL, et al.: Evidence-based guideline for the management of high blood pressure in adults: report from the panel members appointed to the Eighth Joint National Committee [JNC 8]. J Am Med Assoc. 2014, 311:507-520. 10.1001/jama.2013.284427

21. Fryar CD, Ostchega Y, Hales CM, Zhang G, Kruszon-Moran D: Hypertension Prevalence and Control Among Adults: United States, 2015-2016. NCHS Data Brief, no 289. U.S. Department of Health and Human Services, CDC, National Center for Health Statistics, Hyattsville, MD; 2017.

22. Pennant M, Davenport C, Bayliss S, et al.: Community programs for the prevention of cardiovascular disease: a systematic review. Am J Epidemiol. 2010, 172:501-516. 10.1093/aje/kwq171

23. Reaching across the divide: finding solutions to health disparities. (2007). Accessed: July 29, 2018: https://stacks.cdc.gov/view/cdc/12108/.

24. Kong W: Community programs to increase hypertension control. J Natl Med Assoc. 1989, 81:13-16. 